\title{
La cooperación internacional para el desarrollo y la educación superior
}

\author{
International Development Cooperation and higher education \\ Recibido: 13-04-2019 • Aprobado: 28-05-2019 • Página inicial: 45 - Página final: 61 \\ Jorge Enrique Gallego Vásquez*
}

Resumen: el presente artículo hace una refl exión sobre la cooperación internacional, en particular la cooperación sur-sur para el desarrollo a través de la educación superior, con una mirada globalizante, desde la posibilidad de que buenas prácticas con sello colombiano puedan ser apropiadas en otros contextos y viceversa, a nivel latinoamericano o en general, en el sur global.

Palabras clave: cooperación internacional, desarrollo, educación superior, internacionalización.
Abtract: This article makes a reflection on cooperation international, in particular the southsouth development cooperation through higher education, with a globalizing look, from the possibility of good practices with Colombian seal can be appropriate in other contexts and vice versa, at the Latin American level or in general, in the global south.

Keywords: Cooperation international, development, education superior internationalization

JEL: O15

\footnotetext{
* Doctor en Ciencias Pedagógicas, Magíster en Paz, Ciudadanía y Desarrollo, Especialista en Gerencia de Proyectos e Ingeniero y Tecnólogo de Sistemas. Vicerrector Académico de la Corporación Universitaria Lasallista. Profesor de posgrado en diferentes universidades del país. jegv64@hotmail.com ORCID: https://orcid.org/0000-0002-8674-2755
} 


\section{Contexto oulogo,}

\section{Cooperação Internacional para o Desenvolvimento e ensino superior}

Resumo: este artigo faz uma reflexão sobre cooperação internacional, em particular a cooperação sul-sul para o desenvolvimento através do ensino superior, com um olhar globalizante, do possibilidade de boas práticas com selo colombiano pode ser apropriado em outros contextos e vice-versa, no nível latino-americano ou em geral, no sul global.

Palavras-chave: cooperação internacional, desenvolvimento, educação internacionalização superior. 


\section{Introducción}

La educación superior como pilar del desarrollo humano y social y como función social para la incorporación de la Ciencia, la Tecnología y la Innovación en los procesos sociales y organizacionales, tiene retos estratégicos para cumplir con su misión; uno de ellos es la internacionalización, es decir, la capacidad de formar profesionales con pensamiento y acción "glocal", lo que significa pensar globalmente para actuar localmente.

En este sentido el Estado colombiano -hago énfasis en el Estado, no en el Gobierno-, ha establecido a lo largo de las últimas dos décadas cuatro políticas cuyas estrategias, programas y proyectos pueden variar de fondo y de forma, pero que, como tales prevalecen, inspiradas en la declaración de la Conferencia Mundial sobre Educación Superior de la Unesco en 1998, y que se reitera en la del año 2009, cuando se plantea como imperativo "el acceso, equidad y calidad" (Unesco, 2009, p.3). Tales políticas son:

- Cobertura. Política orientada a incrementar el acceso y el éxito académico de los estudiantes a la educación superior, mediante la creación de nuevas Instituciones de Educación Superior (IES) y/o la ampliación del portafolio de programas de pregrado y/o el mejoramiento de las infraestructuras para albergar más estudiantes y/o la incorporación de Tecnologías de Información y Comunicación (TIC) para el incentivo a la educación a distancia y virtual.

- Calidad. Política orientada al mejoramiento continuo de los procesos misionales y de apoyo de las IES, a través del sistema de aseguramiento de la calidad que incluye las pruebas de entrada a la educación superior (Saber 11), las pruebas de salida de la educación superior (Saber Pro), el registro calificado de programas en cumplimiento de condiciones de calidad con carácter obligatorio y la acreditación de alta calidad de programas e instituciones en cumplimiento de factores de calidad con carácter "voluntario".

- Pertinencia. Política orientada a que la oferta de programas de pregrado y posgrado de las IES, corresponda en denominación, justificación, lineamientos curriculares e impacto del graduado en el medio, a las necesidades y oportunidades propias de las regiones, territorios y ciudades donde se ofrecen dichos programas, de tal forma que la oferta no responda solo a la ley del mercado, en función de la demanda de los jóvenes, aún aferrados a las ofertas tradicionales, sino, más bien, a los problemas apremiantes del contexto, donde los futuros graduados interactuarán con las comunidades y las organizaciones públicas y privadas, para aportar con soluciones innovadoras que contribuyan al desarrollo local y regional. 


\section{Contexto Gallego,}

- Internacionalización. Política orientada a promover en las comunidades académicas de las IES colombianas una dinámica de interacción con conocimientos científicos y tecnologías de frontera, los cuales generalmente se producen en países desarrollados, pero también en países en desarrollo donde el intercambio de experiencias permite brindar a estudiantes, profesores y administrativos una visión global de las realidades, necesidades y alternativas de solución a problemas locales, mediante la transferencia de buenas prácticas, tal como lo propone la declaración mundial sobre educación superior en cuanto a "internacionalización, regionalización y mundialización” (Unesco, 2009, p.5).

La internacionalización como política de estado que, a través del Ministerio de Educación Nacional de Colombia (MEN), propende por la incorporación de acciones que permitan que las instituciones de educación superior (IES) tengan un contacto permanente con el contexto global, se concentra en tres estrategias:

1. Internacionalización del currículo. Mediante la apropiación del conocimiento de frontera en el ámbito internacional, relacionado con los contenidos, métodos y medios didácticos para la oferta de cada uno de los programas, sus disciplinas docentes, módulos o cursos, lo que incluye, por ejemplo, bibliografía, materiales pedagógicos, laboratorios, sistemas y tecnologías de información, entre otros.

2. Manejo de idiomas. Mediante la incorporación curricular o extracurricular de al menos un segundo idioma, que en nuestro medio generalmente es inglés; para que, según el Marco Común Europeo, se puedan certificar competencias de docentes y estudiantes, al menos en nivel intermedio (B1).

3. Movilidad académica. Mediante los convenios de intercambio académico con universidades extranjeras, principalmente de profesores y estudiantes, que van y vienen, generalmente en facultades y programas afines, donde se hace reconocimiento de cursos y créditos académicos cursados, o se brinda incluso doble grado académico (mismo título profesional de dos IES distintas) o mediante el desarrollo de proyectos de investigación o proyección social para fortalecer relaciones y redes de conocimiento en el ámbito internacional.

Si bien la inmensa mayoría de las IES cuentan con una Oficina de Relaciones Internacionales (ORI), dedicada a trabajar en estos temas e integrar las acciones de la institución en sus diferentes unidades académicas o facultades; todo el esfuerzo se orienta a beneficiar su comunidad académica en Colombia, aun cuando se espera que los intercambios de profesores y estudiantes extranjeros generen impacto en sus universidades; la medición de indicadores generalmente 
está orientada al impacto en Colombia, precisamente para mostrar cómo la IES gestiona su internacionalización, desde esa perspectiva.

A continuación, se hace una reflexión sobre la cooperación internacional para el desarrollo y la internacionalización institucional que se logra mediante la apertura de IES en otros países a través de la cooperación sur-sur, a partir de la experiencia de "Transferencia del modelo Uniminuto a Costa de Marfil en África del Oeste, para la creación de la Institución Universitaria Tecnológica Eudista de África”.

\section{Sobre el concepto de desarrollo}

Desarrollo en su concepción tradicional, aparece por primera vez en el discurso de posesión del presidente Harry Truman (1949) cuando estableció como premisa de dominación que EE. UU. era un país desarrollado y que los países del Sur global eran países subdesarrollados, lo que convertía a su modelo de desarrollo en un ejemplo a seguir y único camino para el progreso de los países "pobres", no obstante, detrás de la idea de contribuir al "desarrollo económico de los países subdesarrollados" (Escobar, 2007, p.20), también estaba el objetivo de desplegar un modelo socioeconómico que hoy impera en el planeta y que todos conocemos como capitalismo.

El concepto de desarrollo en una sociedad está asociado, en primera instancia, a la satisfacción de necesidades básicas, tales como la salud, la educación, la vivienda, el vestido y la alimentación. Sin embargo, este concepto, alineado con la economía, se desvirtuó en función del crecimiento económico, que no siempre se asocia al desarrollo social y beneficia a los dueños de los grandes capitales financieros nacionales e internacionales, genera más inequidad y mantiene brechas infranqueables entre ricos y pobres en todo el planeta.

Posteriormente, y ante la presión de los movimientos sociales medioambientales, feministas y pacifistas, por el carácter patriarcal, extractivo y consumista del modelo socioeconómico imperante, se propone desde la periferia del sistema mundo un modelo de posdesarrollo que es una visión de "desarrollos alternativos o alternativas al desarrollo" (Gudynas, 2017), donde la discriminación, la guerra y el consumismo no tienen cabida, especialmente en los países del sur global (Fernández, Lauxmann y Trevignani, 2014). Este concepto hace referencia a los países de Latinoamérica, África y Asia, que tradicionalmente se han reconocido como subdesarrollados o en vía de desarrollo, pero que actualmente están construyendo su identidad desde la diversidad, y a través de la cooperación sur-sur intercambian cultura, conocimientos y experiencias en forma de buenas prácticas para resolver problemas comunes, pero de manera contextualizada. 


\section{Contexto oulogo,}

\section{Sobre la noción de súper-desarrollo}

Para repensar un sistema mundo donde los seres humanos podamos satisfacer nuestras necesidades básicas con equidad y justicia social, donde la producción y el consumo responsables garanticen que los recursos se renueven para garantizar la vida de las generaciones futuras en el planeta Tierra; más allá del desarrollo y el posdesarrollo, se plantea un esquema de súper-desarrollo, en referencia a un desarrollo superior, el desarrollo de la madre tierra, nuestra casa común (Papa Francisco, 2015), donde no solo se incluya el desarrollo del ser humano, sino también el desarrollo del planeta mismo sin interferir y desequilibrar sus ciclos vitales naturales. Este concepto, que tímidamente desarrollé en mi tesis de maestría en paz, ciudadanía y desarrollo, cada vez lo veo más claro y a la vez más utópico ante la realidad prácticamente irreversible del daño ecológico que estamos provocando hoy y que hacia el futuro no se avizoran verdaderos cambios que demuestren lo contrario.

El súper-desarrollo es una necesidad y una obligación de la cual depende la vida de nuestra especie en el planeta; un desarrollo superior a la soberbia humana de creerse la especie dominante que cosifica la naturaleza, sintiéndose su dueño y convirtiéndola en un "recurso" o un "insumo" para sus fines consumistas. Súper-desarrollo que suena utópico cuando se observa cómo el poder de los grandes capitales internacionales desarrolla proyectos aeroespaciales para convertir a la especie humana en interplanetaria, buscando la "colonización" de lugares como la Luna o Marte ante la inminencia del destino fatal que tenemos en el planeta azul si seguimos como vamos.

\section{Cooperación sur-sur para el desarrollo}

La definición de cooperación internacional está asociada con el apoyo que un país con mayor nivel de desarrollo le brinda a otro país con menor nivel de desarrollo y que prosperó a partir de la conceptualización del desarrollo como modelo occidental asociado al progreso, a través del avance de la industrialización y la incorporación de tecnologías en todas las dimensiones de la vida de las comunidades, para lo cual, por supuesto, es necesario un sistema político basado en la democracia y el respeto por los derechos fundamentales de los seres humanos. Tal como lo propone la Red Nacional de Agencias de Desarrollo Local de Colombia (Red Adelco) en su sitio web:

Se entiende por Cooperación Internacional la acción conjunta para apoyar el desarrollo económico y social del país, mediante la transferencia de tecnologías, conocimientos, experiencias o recursos por parte de países 
con igual o mayor nivel de desarrollo, organismos multilaterales, organizaciones no gubernamentales y de la sociedad civil. También se conoce como cooperación para el desarrollo y es un concepto global que comprende diferentes modalidades de ayuda que fluyen hacia los países de menor desarrollo relativo. La cooperación internacional o las Ayudas Oficiales al Desarrollo (AOD) se enmarcan en dos grandes criterios: los intereses del donante y las necesidades del receptor o país socio, pero el fondo de la misma se orienta a menguar la brecha de la pobreza en los países a través del fortalecimiento de sus gobiernos locales, la asociatividad, el mejoramiento de la productividad, el intercambio de experiencias o tecnología, entre otras actividades o programas (Red Adelco, s.f.).

Sin embargo, desde un punto de vista crítico, la cooperación internacional, en la misma línea del discurso tradicional de desarrollo, ha movilizado a lo largo de las últimas décadas del siglo XX y hasta ahora, recursos financieros de los países desarrollados, principalmente del norte, hacia países en desarrollo, principalmente del sur; generando problemas de dependencia, asistencialismo e influencia ideológica, política y cultural, con el objetivo de extender el poder de los capitales financieros, que definen a los países en desarrollo como "mercados emergentes", para ampliar la comercialización de bienes y servicios y aprovechar los recursos naturales de estos países que, en su mayoría, viven de economías extractivas y agrícolas, donde la industrialización derivó en maquilas para garantizar costos de producción más bajos y mano de obra más barata. Por lo anterior, la cooperación internacional para el desarrollo debe tener en cuenta la contextualización de las soluciones a las problemáticas locales y los conocimientos y experiencias de las comunidades en clave de súper-desarrollo, para proteger todas las formas de vida y el uso sostenible de los recursos naturales y evitar que se pierdan las culturas ancestrales de los países llamados del tercer mundo, que corresponden a la fábula de los tres mundos que plantea Escobar (2007).

- Primer mundo. El mundo occidental, industrializado, avanzado tecnológicamente, modelo de desarrollo con liberalismo y democracia Neoliberal, economía de mercado. Representado por EE. UU., Gran Bretaña, Francia y en general la Unión Europea.

- Segundo mundo. El mundo socialista y comunista, con desarrollo industrial y tecnológico opuesto a la ideología occidental, dictatorial, monopartidista, economía planificada. Representado por la ex Unión Soviética, China, Corea del Norte, un mundo en extinción. 


\section{Contexto Gallog,}

- Tercer mundo. El mundo de los países subdesarrollados, pobres, con democracias incipientes, en un plano de inferioridad y dependencia. Representado principalmente por países de Latinoamérica, África y Asia, convertidos en el segundo mundo por el colapso del socialismo.

La cooperación tradicional se ha dado en el esquema norte-sur por razones obvias. La cooperación norte-sur o vertical, tal como lo plantea la Agencia Presidencial de Cooperación, es "aquella que se da entre un país desarrollado y otro en vía de desarrollo o de menor nivel de desarrollo" (APC Colombia, s.f.).

La cooperación, según la fuente de recursos técnicos o financieros, puede ser ayuda oficial al desarrollo cuando es patrocinada por organismos oficiales o cooperación privada cuando son entidades privadas que a través de fundaciones y ONG movilizan recursos humanos, técnicos y financieros. Por otro lado, según la relación entre los países y entidades se habla también de cooperación horizontal (sur-sur) o cooperación triangular, la cual se ve apoyada por algún país desarrollado o de primer mundo.

La cooperación se expresa mediante proyectos que responden a necesidades o problemáticas del país beneficiario. La mayoría de los proyectos se han orientado a la solución de problemas asociados a la pobreza extrema, donde el saneamiento básico, la alfabetización y la educación básica, la salud, y los problemas políticos y conflictos bélicos han acaparado los recursos de apoyo a estos países. Sin embargo, donde la cooperación puede contribuir de manera decidida a la superación de la pobreza extrema es en materia de educación superior e investigación, tal como lo plantea la Unesco.

Los establecimientos de enseñanza superior del mundo entero tienen la responsabilidad social de contribuir a reducir la brecha en materia de desarrollo mediante el aumento de la transferencia de conocimientos a través de las fronteras, en particular hacia los países en desarrollo, y de tratar de encontrar soluciones comunes para fomentar la circulación de competencias y mitigar las repercusiones negativas del éxodo de competencias (Unesco, 2009, p.5).

La ignorancia y la pobreza constituyen la esclavitud del siglo XXI, la educación es el camino para la comprensión de la realidad y la formación humana y social que permita la "preservación" de la cultura y la solución de los problemas apremiantes que aquejan a la humanidad; sin embargo, la cultura que constituye el conocimiento colectivo de una sociedad creado para la preservación de la vida, hoy no cumple su misión, ante la gravedad de los efectos del cambio climático, 
el calentamiento global, el consumismo y la utilización indiscriminada de recursos naturales no renovables para sostener las economías del planeta; por lo que se hace necesario repensar la cultura y transformar el sistema económico que desde la formación de las nuevas generaciones cambie el paradigma tradicional de desarrollo y contribuya a la apropiación de hábitos de consumo responsable y protección medioambiental.

Tal como se planteó ya, la internacionalización en el contexto local de las IES se orienta a que las comunidades académicas se desarrollen con una visión global de la realidad, pero con una orientación a la transformación de su entorno mediante la contextualización del conocimiento para contribuir a la solución de problemas sociales y organizacionales. Por otro lado, la transferencia de conocimientos a través de las fronteras hacia los países en desarrollo supone que ese conocimiento proceda de países con mayor nivel de desarrollo, pero con los mismo problemas, lo que configura la cooperación sur-sur, en la que se comparten buenas prácticas o experiencias exitosas y comprobadas en contextos con características similares.

Existen muchas experiencias de internacionalización de la educación superior que, más allá de la internacionalización académica tradicional, trascienden las fronteras de sus países de origen, como el caso de ILUMNO o Whitney International University System, que conforma una red de universidades que comparten recursos tecnológicos, administrativos y financieros desde EE. UU. para América Latina, o universidades como el ITESM de México, que tiene presencia en América, Europa y Asia con educación en línea, posgraduada y continua a la medida, y en el contexto colombiano, universidades como la UDES, con presencia en Panamá o la UNAD con presencia en EE. UU., entre otras que han trascendido las fronteras de sus países de origen. De hecho, gracias a la educación virtual, todas las IES tienen la potencialidad de llegar a cualquier lugar del planeta a través de la gestión internacional de sus procesos misionales (docencia, investigación y extensión o proyección social).

Por supuesto las experiencias aquí resumidas no representan en sí mismas ejemplo de cooperación sur-sur sino, más bien, estrategias de expansión organizacional en el marco de la internacionalización y las oportunidades de ampliación del mercado que esta internacionalización representa. Para terminar esta reflexión, a continuación se aborda la experiencia de la Corporación Universitaria Minuto de Dios, a través del proyecto de "Transferencia del modelo Uniminuto en Colombia a la Congregación de Jesús y María-CJM- en Costa de Marfil, para la creación de la Institución Universitaria Tecnológica Eudista de África". 


\section{Contexto suleo, ,}

\section{La experiencia de Uniminuto en África}

\section{Antecedentes}

La Corporación Universitaria Minuto de Dios (Uniminuto), con casi tres décadas de vida académica, nace de la obra Minuto de Dios en Colombia, fundada por el sacerdote eudista Rafael García Herreros, con 64 años de trabajo por las comunidades más necesitadas en líneas tales como la vivienda, el trabajo comunitario, la educación preescolar, básica, media y superior, el apoyo al emprendimiento y fortalecimiento de unidades productivas, el desarrollo rural y el medio ambiente y por supuesto la evangelización.

Uniminuto, alineada con las políticas de estado en materia de educación superior, es la más pública de las IES privadas, al brindar acceso universal con opción preferencial a las comunidades más deprimidas, a través de estrategias exitosas de regionalización tales como:

- El carisma de la obra Minuto de Dios en Colombia y la espiritualidad eudista reconocida a nivel nacional e internacional.

- Una oferta diversificada de programas en varias áreas de conocimiento, según las necesidades y potencialidades de las regiones donde hace presencia.

- Una oferta de programas en diferentes niveles de formación técnica, tecnológica, profesional universitaria y de posgrado (especializaciones y maestrías).

- Una oferta académica en diferentes modalidades, según las posibilidades de la población objetivo, en metodología presencial, a distancia o virtual.

- Un sistema de financiación a través de la Cooperativa Uniminuto con microcréditos para educación superior que facilitan el acceso, además de precios asequibles.

- Un modelo educativo y curricular que propende por la formación integral con un enfoque de desarrollo humano con responsabilidad social en todos sus programas.

- Un esquema de gestión con una rectoría general y servicios integrados que atienden las necesidades de las cinco rectorías y siete vicerrectorías generales en todo el país, de las cuales se desprenden a su vez, centros regionales y de tutoría en 70 puntos de atención en todo el territorio nacional. 
Gracias a estos factores de éxito, Uniminuto acompañó en Colombia dos IES que en su momento presentaban dificultades, tales como la Fundación Universitaria de Popayán y la Universidad Católica Lumen Gentium de Cali, a las cuales se les transfirió el modelo de gestión y se las acompañó por espacio de 10 años para hacerlas solventes, autosuficientes y con impacto en sus regiones; ambas instituciones prestan hoy un gran servicio para el desarrollo de sus ciudades y áreas de influencia.

De igual forma, Uniminuto obtuvo entre los años 2011 y 2013 reconocimientos internacionales por parte de la Corporación Financiera Internacional (IFC), el G 20 (el grupo de las economías más fuertes del planeta) y el Financial Times, por su modelo incluyente orientado a la base de la pirámide, gracias a cuatro criterios que determinaron el inicio de la experiencia en África:

- Impacto. El impacto social que mejora las condiciones de vida de su comunidad académica, con inclusión social de estudiantes de bajos recursos.

- Innovación. El uso intensivo de sistemas y tecnologías de información en sus procesos académicos y administrativos.

- Sostenibilidad. La gestión financiera que garantiza con precios asequibles, excedentes para la inversión en el desarrollo de la institución y su comunidad.

- Replicabilidad. Un modelo de gestión y de educación escalable que puede ser replicado en otros contextos con similares condiciones.

\section{La sistematización del modelo}

Con base principalmente en el criterio de Replicabilidad, se planteó la posibilidad en 2012 de llevar el modelo Uniminuto a otros países en desarrollo, lo cual quedó plasmado en el plan de desarrollo 2013-2019, en el reto de "Alianzas e Internacionalización". Esta posibilidad se concretó gracias a que el padre Camilo Bernal, en ese momento superior general de la CJM en Roma, visitó Costa de Marfil y Benín, dos países del África del Oeste donde hay presencia eudista desde hace 60 años, e identificó la posibilidad de desarrollar un proyecto educativo como el de Uniminuto.

Fue así como en el año 2013 se hicieron las primeras misiones de intercambio académico y cultural a ambos países para establecer la viabilidad de la propuesta y se firmó un convenio de cooperación interinstitucional para tal efecto. 


\section{Contexto oulogo,}

Sin embargo, la transferencia del modelo Uniminuto suponía la existencia de un modelo documentado, lo cual en ese momento no estaba tan claro. Si bien la institución, con base en el sistema de aseguramiento de la calidad del Ministerio de Educación Nacional (MEN), contaba con documentos institucionales que hacían explícito parte del conocimiento organizacional, tales como el Proyecto Educativo Institucional (PEI), el Plan de Desarrollo, el mapa de procesos y el sistema de información de procesos, procedimientos y formatos para el Sistema de Gestión de Calidad (SGC), al igual que los documentos maestros de los programas académicos, no existía un documento integral con toda la información del modelo y sus componentes.

Fue así como entre 2014 y 2015 se desarrolló el proyecto de sistematización del modelo integral Uniminuto, con el apoyo de una empresa española experta en gestión de conocimiento llamada ICA2. En un trabajo de construcción colectiva de conocimiento se elaboró el esquema general del modelo, se identificaron tres capas (Identidad, Operación y Relacionamiento) con sus respectivos componentes, los cuales a su vez derivaron en módulos conceptuales e instrumentales, y estos últimos se tradujeron en 75 prácticas que recogen el "how to do" de la organización en la capa de operación, lo que permitiría hacer una transferencia más efectiva.

En su construcción participamos 18 personas entre sacerdotes, rectores, vicerrectores y líderes de los procesos misionales y de apoyo, liderados por el presidente de la Organización Minuto de Dios, padre Diego Jaramillo y el rector general del Sistema Universitario Uniminuto en ese momento, Dr. Leonidas López. El compromiso de la alta gerencia fue factor crítico para el logro de este propósito.

La construcción del modelo en un esquema de componentes, módulos y prácticas permitiría su transferencia e implementación de manera gradual y contextualizada, acorde con los requerimientos y las realidades del país de destino.

El 16 de marzo de 2016 Uniminuto obtuvo el registro de propiedad intelectual y derechos de autor número 10-566-362 del "Manual del sistema modular UNIMINUTO", como obra colectiva e inédita del ámbito científico-técnico, para la protección del conocimiento organizacional allí condensado.

\section{La transferencia del modelo}

La transferencia de conocimientos es la práctica más común en el marco de los proyectos de cooperación sur-sur, donde dos países en desarrollo comparten 
su experiencia en forma de buenas prácticas para contribuir a la solución de problemas comunes. La cooperación norte-sur se caracteriza por la colocación de recursos financieros y en ella el financiador generalmente pone las condiciones y los métodos para el desarrollo de los proyectos, lo que dificulta la implementación y la flexibilización para ajustarse al contexto donde se desarrolla.

Teniendo en cuenta que los recursos de países en desarrollo son limitados para la cooperación, en la figura de asistencia técnica como oferta de cooperación de un país hacia otro que demanda cooperación, son las personas que poseen la experiencia las que hacen la cooperación; por eso, como lo plantea la embajadora de Colombia en Ghana, para África del Oeste, Claudia Turbay Quintero, "la cooperación sur-sur es en esencia un intercambio humano, donde pequeñas acciones pueden producir grandes impactos".

La transferencia inició con el estudio de factibilidad elaborado con el apoyo del IFC y financiado por el gobierno de Cataluña, que en 2014 arrojó resultados favorables para el inicio del proyecto de creación y puesta en marcha de la Institución Universitaria Tecnológica Eudista de África (IUTEA).

Posteriormente se iniciaron los acercamientos con ambos gobiernos, para configurar un proyecto de cooperación sur-sur, y en 2015, con respuesta positiva de la oficina de oferta de cooperación de la Agencia Presidencial de Cooperación Internacional de Colombia (APC-Colombia), se iniciaron misiones de trabajo en las que viajamos a Costa de Marfil como "embajadores de conocimiento" con el fin de hacer los estudios jurídicos para la creación de una universidad en este país. De la misma manera, el gobierno marfileño, a través de su primer ministro, hoy vicepresidente de la república, el señor Daniel Kablan Duncan, dispuso el acompañamiento del Ministerio de Educación Superior e Investigación Científica para surtir todos los trámites legales y cumplir con la normativa para la creación y puesta en marcha de la institución. El relacionamiento se convirtió así en factor crítico de éxito luego de que una delegación del gobierno marfileño visitara Colombia por invitación del MEN y Uniminuto, con el apoyo de la Cancillería, para conocer el sistema educativo colombiano y el modelo Uniminuto.

Un proyecto de universidad que contribuya a incrementar la cobertura de la educación superior en un país como Costa de Marfil donde el 38\% de los jóvenes terminan el bachillerato y solo el $12 \%$ ingresan a la educación superior, que en el año 2014 solo tenía 200.000 estudiantes en las universidades públicas y privadas, con un esquema francés de educación pública y gratuita insostenible, 


\section{Contexto oulogo,}

según el estudio de factibilidad (Descarrega, 2014), se convirtió en un desafío y una oportunidad.

Por supuesto, en atención a que los recursos de Uniminuto y de la CJM en África eran limitados y cubrían parte de los desplazamientos y la estadía, el grueso de la inversión era la infraestructura y la dotación, por lo que el proyecto para la construcción del primer centro de formación en la comuna de Yopougon en la ciudad de Abijan, se presentó a diferentes agencias internacionales de cooperación, y finalmente la Conferencia Episcopal Italiana (CEI), que maneja un "fondo para el apoyo al desarrollo de países del tercer mundo", correspondiente a un porcentaje de los impuestos del pueblo italiano, aprobó el proyecto, y fue así como en 2016 se inició el proceso de trámite de creación de la IUTEA y de diseño y construcción del edificio en un terreno de los padres eudistas, como contrapartida, incluyendo el trabajo del equipo humano colombo-marfileño implicado en el proyecto. Con el apoyo económico de la CEI se configuró esta experiencia como un proyecto de cooperación sur-sur y triangular, que en septiembre de 2018 fue reconocida como "buena práctica" de cooperación sur-sur y triangular en la categoría Quality Education para contribuir al logro de los Objetivos de Desarrollo Sostenible (ODS) (Naciones Unidas, 2018, p.93).

En diciembre de 2017 se obtuvo la resolución de creación y apertura de la IUTEA por parte del Ministerio de Educación Superior e Investigación Científica de Costa de Marfil.

Se diseñó y ejecutó un curso de formación de formadores en el que 47 de 72 participantes se certificaron en pedagogía, didáctica, currículo y TIC para la educación, los cuales serían candidatos a docentes de la IUTEA.

Se hizo la adecuación curricular de cuatro programas de Uniminuto para convertirlos en la oferta de licenciatura y máster de la IUTEA, con base en el modelo educativo marfileño; son ellos: Administración de Empresas, Ingeniería Civil, Ingeniería Informática e Ingeniería Agroecológica, los cuales responden a las necesidades identificadas en los sectores estratégicos de desarrollo de Costa de Marfil.

El 14 de noviembre de 2018 se inauguró el edificio San Juan Eudes, primera sede de la IUTEA en la comuna de Yopougon, y se iniciaron actividades académicas con tres sacerdotes eudistas en la dirección del centro, siete colaboradores en las áreas académicas y administrativas, 17 profesores y 47 estudiantes, así como actividades de proyección social mediante tres talleres con comunidades 
en el área de agricultura urbana con 250 beneficiarios, y dos conferencias, cada una sobre pedagogía y didáctica de la educación superior y la práctica profesional en Colombia como estrategia de relación Universidad-Empresa, dirigidas a 50 profesores, directivos y funcionarios públicos del Ministerio del Empleo y la Formación Profesional y de la Cámara de Comercio e Industria de Costa de Marfil.

Los desafíos futuros son hacer sostenible la operación incrementando el número de estudiantes con el apoyo del gobierno marfileño a través de estudiantes becados, como también del mercadeo y el branding de la nueva institución, y mediante un programa de fund raising para la financiación de estudiantes de escasos recursos que, por supuesto, son la mayoría de la población.

Dentro del plan de desarrollo 2020-2025 de la IUTEA, y atendiendo a la meta de tener 20.000 estudiantes en 10 años en centros distribuidos en las ciudades más importantes del país, también con educación a distancia y virtual, se contempla la creación de dos centros más en los próximos 2 años: uno en otra comuna de la ciudad de Abijan que, con 7 millones de habitantes, es la más grande del país, y otro en la ciudad de Bouaké, segunda ciudad del país, donde el señor Nanan Koffi, jefe tradicional de la región de Gbeke, donó a la IUTEA un terreno para que se construya allí una nueva sede. Cabe resaltar que Nanan Koffi visitó Colombia y conoció Uniminuto con el apoyo de APC-Colombia en una de las misiones, razón por la cual decidió con su consejo de notables, en reunión que hicimos en noviembre de 2018, apoyar el proyecto bajo la premisa de que en cooperación sur-sur "todos ponen".

\section{Conclusiones}

La cooperación sur-sur es en esencia el intercambio de cultura, conocimientos y experiencias traducidos en buenas prácticas para la solución de problemas comunes entre países con diferentes niveles de desarrollo del sur global.

El desarrollo, como concepto asociado al modelo de sociedad que garantice el bienestar de la población en general y el bien común, debe ser redefinido y resignificado en función de la sostenibilidad del medio ambiente y el consumo responsable de bienes y servicios.

La internacionalización de la educación superior va más allá de desarrollar estrategias para el desarrollo de una cultura planetaria en la comunidad académica en el país de origen de las instituciones y debe trascender las fronteras, primero con la movilidad académica a través de la docencia y la investigación, y luego mediante la transferencia de conocimientos especialmente a comunidades 


\section{Contexto oulogo,}

necesitadas con problemas similares y alternativas de solución ya probadas en el contexto de las IES.

La interacción con comunidades de otros países del sur global es la armonización de culturas, lo que también se conoce como inculturación, basada en la inclusión y la aceptación de la unidad en la diversidad, que hace parte de una realidad generalizada en el planeta Tierra, nuestra casa común.

\section{Referencias}

Agencia Presidencial de Cooperación Internacional (APC Colombia). (s.f.). ¿Cuáles son las modalidades de cooperación internacional? Recuperado de http://www.apccolombia.gov.co/pagina/cuales-son-las-modalidadesde-cooperacion-internacional

Descarrega, S. (2014). Estudio de factibilidad para la creación de una institución universitaria en Costa de Marfil con base en el modelo Uniminuto en Colombia. IFC, Uniminuto.

Escobar, A. (2007). La invención del Tercer Mundo. Construcción y deconstrucción del desarrollo. Serie Colonialidad/modernidad/ descolonialidad. Caracas, Venezuela: El Perro y la Rana Ediciones. Recuperado de http://www.cronicon.net/paginas/Documentos/No.10.pdf

Fernández, V., Lauxmann, C. y Trevignani, M. (2014). Emergencias del Sur Global. Perspectivas para el desarrollo de la periferia latinoamericana. Economia e Sociedade, Campinas, 3(52), 611-643.

Gudynas, E. (2017). Posdesarrollo como herramienta para el análisis crítico del desarrollo. Estudios Críticos sobre el Desarrollo, 7(12), 193-210.

Naciones Unidas. (2018). Buenas Prácticas en la Cooperación Sur-Sur y Triangular para el Desarrollo Sostenible. Volumen 2. Oficina de las Naciones Unidas para la Cooperación Sur-Sur. Recuperado de https:// www.unsouthsouth.org/2018/11/28/good-practices-in-south-south-andtriangular-cooperation-for-sustainable-development-vol-2-2018-inseven-languages/ 
Papa Francisco. (2015). Carta Encíclica Laudato Si. Sobre el cuidado de la casa común. Ciudad del Vaticano.

Red Adelco. (s.f.). ¿Qué es cooperación internacional? Red Nacional de Agencias de Desarrollo Local de Colombia. Recuperado de https://www. redadelco.org/que-es-la-cooperacion-internacional .

Unesco. (2009). Declaración final-Conferencia mundial de educación superior. Las nuevas dinámicas de la educación superior y la investigación para el cambio social y el desarrollo. París. Recuperado de https://www.cna. gov.co/1741/articles-186502_Conferencia_Mundial_2009.

\section{Para citar este artículo:}

Gallego, J. (2019). La cooperación internacional para el desarrollo y la educación superior. En-Contexto, 7(11), 45-61. 